\title{
Le sikhisme en sa diversité : à propos de trois ouvrages récents
}

Denis Matringe

\section{(2) OpenEdition \\ 12 Journals}

Édition électronique

URL : http://journals.openedition.org/assr/26276

DOI : $10.4000 /$ assr.26276

ISSN : $1777-5825$

Éditeur

Éditions de l'EHESS

\section{Édition imprimée}

Date de publication : 31 décembre 2014

Pagination : 29-41

ISBN : 978-2-7132-2467-6

ISSN : 0335-5985

\section{Référence électronique}

Denis Matringe, "Le sikhisme en sa diversité : à propos de trois ouvrages récents ", Archives de sciences sociales des religions [En ligne], 168|2014, mis en ligne le 30 mars 2018, consulté le 19 avril 2019. URL : http://journals.openedition.org/assr/26276 ; DOI : 10.4000/assr.26276

Ce document a été généré automatiquement le 19 avril 2019

(c) Archives de sciences sociales des religions 


\title{
Le sikhisme en sa diversité : à propos de trois ouvrages récents
}

\author{
Denis Matringe
}

\section{RÉFÉRENCE}

Singh Pashaura and Fenech Louis E. (dir.), The Oxford Handbook of Sikh Studies, Oxford, Oxford University Press, 2014, 704 p. (bibliographie, index).

Takhar Opinderjit Kaur, Sikh Identity : An Exploration of Groups among Sikhs, Hants (RU) et Burlington (ÉUA), 2005, xv + 215 p. (glossaire, illustrations, bibliographie, index).

Singh Joginder, The Namdhari Sikhs: Their Changing Social and Cultural Landscape, New Delhi, Manohar, 2013, 216 p. (bibliographie, index).

1 La présente note fait suite à trois autres publiées précédemment au sujet des études sur les sikhs et le sikhisme ${ }^{1}$. Elle traite de trois ouvrages qui forment un ensemble analogue à une poupée russe: le premier, un très complet manuel d'études sikhes, comporte un chapitre sur les sectes sikhes, qui sont l'objet du deuxième, le troisième traitant quant à lui en détail de l'une des sectes succinctement présentées dans le deuxième. Les trois livres seront donc abordés dans cet ordre, qui n'est pas celui de leurs dates de parution respectives.

2 Il existait jusqu'au printemps 2014 de bons livres d'introduction au sikhisme et à l'histoire des sikhs, mais chercheurs, étudiants et personnes intéressées ne disposaient pas d'un manuel qui tout à la fois expose les diverses facettes du sikhisme et fasse le point sur l'état de la recherche les concernant. Cette lacune est désormais comblée, et admirablement, avec The Oxford Handbook of Sikh Studies, dirigé par Louis Fenech, professeur d'études sikhes et d'histoire de l'Asie du Sud à l'Université de l'Iowa du Nord, et Pashaura Singh, professeur, titulaire de la chaire Jasbir Singh Saini à l'Université de Californie (Riverside). Ce livre est venu prendre place dans la prestigieuse collection des Oxford Handbooks, qui couvrent quatorze disciplines réparties entre Humanités, Mathématiques et sciences, Psychologie et neurosciences, Sciences sociales et études 
interdisciplinaires. Le volume consacré aux études sikhes s'insère, à l'intérieur de l'ensemble « Humanités », dans la discipline « Histoire des religions ».

3 L'objectif scientifique qui confère son unité à ce manuel consiste à donner voix aux diverses expressions de la sikhness (« sikhité », sikhī en hindi, ourdou et panjabi), - terme que les directeurs de l'ouvrage et les auteurs de leur équipe préfèrent à celui de «sikhisme» (en raison de ma "francité ", je parlerai toutefois pour ma part, en ce sens, de «sikhisme »). Il en résulte une encyclopédie dynamique et plurielle du monde sikh, dans son histoire et son présent, fondée sur une grande diversité de sources allant des fonds d'archives publics et privés aux résultats d'enquêtes de terrain en passant par des collections muséographiques et des enregistrements de musique et de chant.

4 La mise en chantier d'une telle somme a été rendue possible parce que, depuis le milieu des années 1990, des chaires et des enseignements d'études sikhes et panjabies ont été créées dans plusieurs pays occidentaux. Il existe ainsi désormais huit chaires d'études sikhes et panjabies aux États-Unis d'Amérique (EUA), et sur les quarante-cinq contributeurs du manuel, qui ne sont pas tous professeurs titulaires d'une chaire, dixneuf sont en poste aux EUA, onze au Royaume-Uni, trois au Canada, un en Belgique, un au Japon et un en Suède. C'est là un bon reflet de la géographie mondiale de la recherche sur les sikhs et le sikhisme aujourd'hui.

Que le sikhisme soit la première des grandes religions de l'Inde représentée dans les Oxford Handbooks n'est pas entièrement un hasard : né au début du XvI siècle autour d'un saint poète nommé Nānak (1469-1539), en qui les sikhs voient leur premier Gurū, il est la plus jeune de ces religions, et parce que $75 \%$ des quelque vingt millions de sikhs de l'Inde vivent dans l'État indien du Panjab, dont ils forment environ $60 \%$ de la population, il est la plus localisée. La matière est donc bien plus aisément gérable que pour l'hindouisme, le bouddhisme, le jaïnisme, le christianisme ou l'islam. Mais dans leur introduction, les directeurs du volume évoquent aussi un autre facteur important pour la genèse de leur entreprise. Les premières recherches novatrices entreprises sur les sikhs et le sikhisme à partir des années 1960 ont fait l'objet de virulentes attaques de la part des savants sikhs orthodoxes, surtout durant la période du vif conflit qui opposa des groupes politicoreligieux sikhs au gouvernement indien dans les années 1980-1990 et vit le développement d'un mouvement fondamentaliste et terroriste en leur sein ${ }^{2}$. Nombre de chercheurs sikhs, en Inde et même en Occident, furent menacés, contraints d'orienter leur travail vers des sujets consensuels, voire même physiquement agressés. Nous n'en sommes plus là, et l'oxford Handbook donne la place qui leur revient et à la critique de l'historiographie traditionnelle (c'est-à-dire conforme au récit de l'histoire des sikhs élaboré par le courant dominant du sikhisme), et aux recherches sur la diversité religieuse, sociologique et culturelle des sikhs.

6 Prenant en compte les différentes manières d'être sikh à travers les âges et les lieux, le manuel explore, du sikhisme, les expressions historique, littéraire, idéologique, institutionnelle, artistique, diasporique, et finalement selon le genre et la caste. Chacune de ses grandes sections consiste en un article d'introduction suivi de plusieurs études particulières. Les recoupements sont nombreux, sans nuire en rien à l'ensemble, et les approches, respectées par les coordinateurs de l'entreprise, sont aussi plurielles que l'objet étudié. Tout en cherchant à donner un aperçu de cet excellent manuel, je m'attacherai à dégager les questionnements nouveaux qui en orientent la dynamique. Concernant l'histoire, c'est la nature fluide et polyphonique du passé sikh qui est mise en lumière. Louis Fenech insiste ainsi, dans sa contribution, sur l'influence de l'idéologie 
moghole sur la cour des Gurūs sikhs au XviI ${ }^{e}$ siècle. Au siècle suivant, nous montre Purnima Dhavan, la figure glorieuse du dernier de ces Gurūs, Gobind (1766-1708), fondateur de l'ordre militant du Khālsā (« les Purs »), se construit dans des récits épiques de ses exploits guerriers et devient un important trait d'union culturel entre des chefferies sikhes désormais dispersées. Sunit Singh relie entre elles, à propos du royaume sikh de Lahore (1799-1849), deux thématiques historiographiques en pleine expansion dans les études indiennes : les modalités de la formation d'un État successeur de l'Empire moghol et le développement d'un marché du travail pour les soldats en son sein, ouvrant la voie aux entreprises des colonisateurs britanniques, qui en jouent bientôt pour asseoir leur domination. Navdeep Sikh Mandair revient pour sa part sur le rôle du colonialisme dans la structuration du sikhisme au xix ${ }^{e}$ siècle. Les Britanniques en effet favorisèrent le triomphe de l'affirmation communautariste d'une identité sikhe étroitement définie, contre la vision plus œcuménique d'une culture sikhe amarrée à la nébuleuse hindoue, contribuant ainsi à la naissance d'un ethnonationalisme sikh au début du $\mathrm{xx}^{\mathrm{e}}$ siècle. Concernant l'histoire des sikhs dans l'Inde indépendante, Joginder Singh met l'accent sur le rôle joué par la caste des Jāts, dominante dans le Panjab rural : les Jāts s'emparent de la direction des principales institutions et les mobilisent contre le Parti du Congrès et l'État central, poussant dans le même mouvement les sikhs membres de castes dominées à affirmer leur différence identitaire. L'article d'Ann Murphy qui conclut ce chapitre sur l'histoire introduit une très intéressante dimension réflexive: l'auteur propose une remarquable synthèse de la formation des diverses traditions historiographies sikhes, rapportées tout à la fois aux besoins de la communauté au fil de son évolution et à leurs modèles extérieurs, d'abord hindous et moghols, puis européens.

7 La section consacrée aux "expressions institutionnelles » renouvelle pour sa part la question des formes et des centres de l'autorité religieuse dans le sikhisme. Les premières contributions examinent des institutions telles que le comité de gestion des temples sikhs, et elles en exposent les liens avec l'organisation politico-religieuse appelée Akali Dal qui exprime, à travers une rhétorique religieuse, les intérêts des grands propriétaires terriens sikhs (Kashmir Singh, Amarjit Singh Nihang). Les chapitres suivants se concentrent pour leur part sur les sectes qui contestent selon différents paradigmes les formes d'autorité religieuse que le courant dominant du sikhisme cherche à imposer à l'ensemble du Panth (litt. « voie » de Nānak, terme par lequel est communément désigné l'ensemble de la communauté de ceux qui se disent sikhs). Parallèlement à ce phénomène sectaire fleurissent au Panjab des séminaires et des centres de vie religieuse organisés par des saints personnages dont les disciples se recrutent souvent au sein d'une caste particulière (Opinderjit Takhar et Paramjit Singh Judge). Une dernière contribution étudie les changements que la globalisation introduit dans le sikhisme, tant en Inde que dans la diaspora (Mark Juergensmeyer).

8 Dans la section traitant de l'« expression artistique», des articles consacrés respectivement à la musique (Gurnam Singh), à la peinture et aux arts matériels (NikkyGuninder Kaur Singh, Ann Murphy) et à la vie du Temple d'Or d'Amritsar (Charles $M$. Townsend) voisinent avec, d'une part, une réflexion théorique sur les arts du spectacle et de l'interprétation (cultural performances) en contexte sikh (Michael Nijhawan) et, d'autre part, avec un chapitre consacré à la programmation d'un musée appelé Khalsa Heritage Complex à Anandpur, ville où se tenait la cour de Gurū Gobind à la fin du XVII siècle, au pied des Shivaliks, au nord du Panjab (William J. Gover). Des trois derniers articles de la section, le premier s'intéresse à l'évolution de l'art martial typiquement sikh 
appelé gatkā, qui se pratique avec épée et bâton, et à sa reviviscence au sein de la diaspora (Kamalroop Singh), le deuxième à l'expression du sikhisme à travers des sites de la Toile, des films et des vidéos - le questionnement portant principalement sur les débats identitaires et les usages du passé, ainsi que sur le rapport entre description et prescription (Susan Elizabeth Prill), et le troisième à la relation entre "sikhité » (sikhï) et " panjabité » (panjābiyyat), entre les idéaux religieux sikhs et une culture régionale que les sikhs partagent avec hindous et musulmans, et dont certains traits (machisme, castéisme) contredisent l'enseignement de leurs Gurūs.

9 La section suivante, présentée par Darshan Singh Tatla, traite de la diaspora, dans l'histoire et dans le présent, tout d'abord en Europe continentale (Kristina Myrvold) et aux EUA (Jaideep Singh), puis en Inde même - un cinquième de la population sikhe du pays (Himadri Banerjee), les questionnements portant principalement sur les formes d'organisation et la création d'institutions, et sur les relations avec les sociétés d'accueil et le maintien des liens avec le Panjab. Les trois derniers chapitres innovent en adoptant une perspective transversale. Le premier traite des pratiques transnationales, au premier rang desquelles les remises d'argent opérées par les sikhs de la diaspora vers les familles restées au Panjab (Shinder Singh Thandi). Le deuxième examine les relations entre sikhs «blancs » (gorās) et sikhs panjabis, avec une attention particulière accordée à la question des conversions, aux principales organisations accueillant les convertis - 3HO (pour « Healthy, Happy, Holy Organization », fondée au début des années 1970 aux EUA par un émigrant sikh surnommé Yogi Bhajan) et Sikh Dharma of the Western Hemisphere, une attention particulière étant accordée aux réactions des sikhs du Panjab à ces mouvements et à la prétention des sikhs "blancs » à l'orthopraxie (Verne A. Dusenbery). Quant au troisième article, il cherche à caractériser la revendication d'un État-nation sikh indépendant et théocratique appelé Khalistan par les fondamentalistes des années 1980-1992 comme une critique politique radicale de la République indienne et de la nature de son fédéralisme et de son sécularisme, cette revendication étant en outre envisagée dans sa parenté avec d'autres mouvements de rébellion au sein de groupes déshérités de l'Inde (Cynthia Keppley Mahmood).

Le sikhisme, en ses Écritures et ses textes doctrinaux, prône l'égalité entre hommes et femmes et le rejet du système des castes. Mais les pratiques sont souvent tout autres, et c'est vers les questions de caste et de genre que se tournent les trois chapitres de la section suivante. Le premier étudie les formes prises parmi les sikhs par le rejet de la caste, mettant l'accent sur la formation de structures sociales et de hiérarchies alternatives ainsi que sur l'impact du mouvement de réforme socioreligieuse des années 1890-1920 et sur les effets de la démocratie politique et du développement économique dans l'Inde indépendante (Surinder S. Jodhka). Le deuxième questionne la place faite (laissée? refusée ?) aux femmes, à travers l'histoire des sikhs, dans les rituels, les institutions, les rôles institutionnels et les codes de conduite, ainsi qu'au regard des marqueurs identitaires, comme les cinq symboles des hommes sikhs du Khālsā (les cheveux non coupés, le peigne, le poignard, le bracelet métallique et le caleçon), tout en tenant compte des variables de région, de classe et de caste (Doris Jakobsh). Le chapitre qui conclut la section est une tentative originale par Nikky-Guninder Kaur Singh, sur la base de ses recherches antérieures, pour proposer une interprétation féminine des Écritures sikhes en matière de théologie, de spiritualité, de vie sociale, de conscience historique et d'identité personnelle, l'auteur préconisant pour les femmes et pour les hommes une égalité d'accès aux textes sacrés et voyant en ces derniers une ressource 
importante pour promouvoir l'action de travailleurs sociaux ainsi que de politiciens et de juristes désireux de promouvoir l'égalité entre les sexes.

11 Dans l'unique article de la section de conclusion, l'un des directeurs du volume, Pashaura Singh, s'interroge sur l'orientation à venir des études sikhes. Il se fait l'avocat des apprentissages linguistiques et du travail sur les textes - de la quête de manuscrits aux traductions - ainsi que de la nécessité de continuer à œuvrer contre les restrictions que cherche encore à imposer à la recherche, en Inde notamment, l'orthodoxie conservatrice.

Ce manuel, dont les chapitres, tout à la fois, offrent un bilan des connaissances acquises et font le point sur les recherches et les questionnements en cours, est appelé à être pour longtemps l'ouvrage de référence dans le champ des études sur les sikhs et le sikhisme.

Les sectes sikhes, dont Opinderjit Kaur Thakar dresse un panorama dans la section «Expressions institutionnelles » du manuel, sont l'un des domaines sensibles des études sikhes sur lesquels la recherche n'avait guère décollé avant les années 1980. La première synthèse scientifique sur le sujet, parue en 2005, Sikh Identity: An Exploration of Groups among Sikhs, est précisément l'œuvre de la même Opinderjit Kaur Thakar, professeur de sciences religieuses à la Heathland School de Hounslow.

Dans l'introduction de son livre, elle revient sur les raisons d'une percée aussi tardive. Comme les autres religions autochtones de l'Inde, le sikhisme réagit par la réforme à l'impact colonial et au défi de l'entreprise missionnaire chrétienne. Religion à prétention universelle issue à partir du début du $\mathrm{XVI}^{\mathrm{e}}$ siècle de l'hindouisme, le sikhisme connut la réforme plus tard que l'hindouisme et l'islam indien, le Panjab n'ayant été conquis par les troupes de Compagnie britannique des Indes qu'en 1849. L'un des traits fondamentaux de la réforme du sikhisme, qui se manifesta à travers la création d'associations appelées Singh Sabhās (Sociétés des Lions), fut l'apparition d'un courant prônant une identité séparée de l'hindouisme et un retour aux sources et aux pratiques originelles. Ce courant, opposé à celui, dit sanātana, des tenants de l'inscription dans la vaste nébuleuse hindoue d'un sikhisme pluriel, prit le nom de Tat Khālsā ou «Vraie communauté des Purs». Implanté d'abord à Lahore et rassemblant une bonne partie de l'intelligentsia sikhe ayant reçu une éducation à l'européenne, ce courant mena une lutte acharnée pour faire accepter par nombre de sikhs et par les Britanniques son interprétation exclusiviste de la foi sikhe et son affirmation du sikhisme comme radicalement séparé de l'hindouisme. À grand renfort de publications, de créations d'écoles et de prédication, il finit par l'emporter et par recevoir l'appui des autorités britanniques, qui exigèrent des sikhs qu'elles enrôlaient dans leur armée indienne qu'ils passent par un rituel colonial d'affirmation de leur foi et qu'ils arborent les symboles du Khālsā. Les dirigeants du Tat Khālsā entreprirent aussi de rédiger un code sikh, finalement publié en 1950 sous le titre de Sikh rahit maryāa $\bar{a}$ (Code de conduite sikh) et comportant une définition du sikh qui se veut définitive et universelle. L'existence de ce code et de cette définition a contribué, affirme l'auteur, à diviser les sikhs qu'il entendait rassembler sous une bannière unique. En effet, le code exige des sikhs qu'ils soient « initiés dans le Khālsā » (ammritdhārīs) et en arborent les symboles, à commencer, pour les hommes, par les cheveux et la barbe non coupés et le port d'un turban. Or, souligne Opinderjit Kaur Thakar, la majorité des sikhs ne sont pas ammritdhārīs. Sur la base de ce constat, son livre est une enquête sur les "groupes sikhs» qui, tout en affirmant, souvent depuis une période ancienne, une identité sikhe, ne peuvent se reconnaître dans les propositions du Sikh rahit maryādā. L'histoire qui vient d'être rappelée est reprise dans le premier chapitre de l'ouvrage, qui examine la question de l'identité sikhe dans une perspective historique et se termine par 
un questionnement de l'autorité suprême au sein du Panth. Un point commun des cinq groupes que l'auteur étudie dans la suite de son livre est précisément qu'ils ne reconnaissent pas l'autorité des institutions mises en place par les membres du Tat Khālsā. Ces cinq groupes sont le Gurū Nānak Nishkām Sevak Jathā, les Nāmdhārīs, les Ravidāsīs, les Vālmīkīs et le Sikh Dharma of the Western Hemisphere. La méthodologie adoptée par l'auteur consiste à examiner ces cinq groupes au regard de cinq critères : sont-ils initiés dans le Khālsā et se conforment-ils au Sikh rahit maryādā ? Quelle est pour eux l'importance de l'Ādi Granth, livre sacré des sikhs compilé en 1604 par leur cinquième Gurū, Arjan (1563-1606), et des dix Gurūs qui se sont succédé à la tête du Panth entre le début du Xvi ${ }^{e}$ siècle et 1708 ? Eu égard à leur foi et à leurs pratiques, chacun de ces groupes peut-il être défini comme hindou, comme sikh, ou comme ni l'un ni l'autre? Quelle est l'attitude de chacun par rapport à la caste, et chacun a-t-il un lien privilégié avec une caste particulière?

Les cinq chapitres suivants sont consacrés à cette enquête, chaque groupe étant aussi présenté dans son histoire. Il serait d'autant plus hors de propos de résumer chacun de ces chapitres que d'une part leur matière est synthétisée par l'auteur dans sa contribution au Handbook présenté ci-dessus, et que d'autre part l'une des sectes étudiées, celle des Nāmdhārīs, fait l'objet du troisième livre abordé dans la présente note. Je voudrais par contre évoquer la vision d'ensemble qui ressort des analyses d'Opinderjit Kaur Thakar et revenir, à partir du chapitre de conclusion, sur certains des thèmes qui parcourent tout le livre.

Deux importants aspects du sikhisme sont mis en évidence par les analyses de l'auteur. En premier lieu, Sikh Identity apporte une contribution décisive à la construction d'une identité sikhe plurielle (l'auteure parle d'« identité fédérale du Panth», p. 189). Il y a dans le Panth des sikhs (originaires) du Panjab et des sikhs d'origine occidentale (des sikhs gore , «blancs »); des sikhs initiés dans le Khālsā, d'autres qui ne le sont pas, et certains hommes, parmi ces derniers, qui arborent néanmoins les symboles du Khālsā; des sikhs du Khālsā qui ne reconnaissent que les dix premiers Gurūs, de Nānak à Gobind, d'autres qui sont les disciples de Gurūs vivants aux lignées plus ou moins anciennes, et d'autres encore, initiés ou non dans le Khālsā, qui sont les fidèles de prédicateurs charismatiques appelés sants (litt. "authentiques»); des végétariens et des non-végétariens; des convertis récents, souvent de basse caste, et des sikhs se proclamant fièrement descendants de l'élite de haute caste de la première communauté ; etc.

Mais une autre distinction doit être établie. Tandis que les membres du Gurū Nānak Nishkām Sevak Jathā, les Nāmdhārīs et les Nirañkārīs affirment leur identité sikhe, Ravidāsīs et Vālmīkīs, qui viennent de castes d'intouchables méprisées par les autres sikhs en dépit des injonctions des Écritures et du Sikh rahit maryādāa, s'en démarquent. Les Ravidāsīs - principalement des camārs, " corroyeurs ", qui donnent, à l'intérieur de l'Ādi Granth, la précellence au saint poète du XVI $\mathrm{e}^{\mathrm{e}}$ siècle. Ravidās, lui-même corroyeur à Bénarès - revendiquent désormais une identité religieuse autonome, ni sikhe ni hindoue. Ils ont leurs propres lieux de rassemblements autour de prédicateurs charismatiques et leurs propres gurdwaras. De leur côté, les Vālmīkīs - essentiellement des cúhrās, «balayeurs », tout en gardant une certaine révérence pour l'Ādi Granth, ont adopté comme texte doctrinal le Yoga-vāsisțha. Ce texte hindou composite sur la libération spirituelle, compilé aux environs des $\mathrm{x}^{\mathrm{e}}$-XII ${ }^{\mathrm{e}}$ siècles, est attribué à Vālmīki, auteur de la grande épopée indienne du Rāmāyaṇa, dont le noyau originel pourrait dater des $v^{\mathrm{e}}$-Iv $\mathrm{v}^{\mathrm{e}}$ siècles av. n.-è., et 
personnage éponyme des Valmīkīs. Dans la vie religieuse de l'Inde du Nord, les Vālmīkīs voisinent harmonieusement avec les fraternités de Sādhūs hindous.

Parallèlement à ces considérations, l'auteur revient régulièrement à de grands thèmes religieux ou sociaux, comme le concept de Gurū, qui n'a plus grande signification pour les Ravidāsīs et les Vālmīkīs, les maîtres et les fondateurs (chaque secte a les siens), l'attitude par rapport au Sikh rahit maryādā, dont le Sikh Dharma of the Western Hemisphere reconnaît l'autorité et dont les Nāmdhārīs ont élaboré leur propre version, et, enfin, l'ethnicité panjabie: cruciale, à cet égard, est la notion d'izzat (de l'arabe 'izzat). Cette dernière renvoie tout à la fois à l'honneur, au respect de soi et au prestige. C'est au nom de l'izzat que les enfants sont poussés par leur parents à accepter des mariages arrangés à l'intérieur de leur caste, quelle que soit la secte à laquelle ils appartiennent - avec un homme de statut égal ou supérieur pour une femme, et avec une femme de statut égal ou inférieur pour un homme. Mais l'ethnicité, c'est aussi la langue panjabie en ses multiples dialectes, et l'attachement des sikhs du Panjab à leur langue, qu'ils utilisent comme langue de liturgie et de prédication, compte dans leurs difficultés à admettre les sikhs «blancs » parmi eux.

Sikh Identity est d'une grande richesse et remplit parfaitement la mission que lui assigne son auteur : donner à voir le Panth en sa diversité. Mais pour le chercheur intéressé par la sociologie des religions de l'Inde, il offre aussi un très riche matériau pour étudier le lien entre secte et caste, surtout si l'on songe que les Jāts du Panjab, tous sikhs et formant près de 50 \% du Panth, adoptent, qu'ils soient initiés ou non, les symboles du Khālsā et le Sikh rahit maryādā (ou sa version nāmdhārī) et que la plupart des sikhs qui ne sont ni initiés dans le Khālsā ni membres d'une secte, et que l'on désigne comme sahajdhārīs (« partisans de l'extase », ou, selon les sikhs du Khālsā qui les méprisent, «tenants de la facilité »), viennent des castes commerçantes et professionnelles des villes, comme celle des Khatrīs. La sociologie est précisément la discipline qui structure le livre de Joginder Singh, The Namdhari Sikhs: Their Changing Social and Cultural Landscape, consacré à l'une des sectes présentées par Opinderjit Kaur Thakar. Les Nāmdhārīs sont aussi appelés Kūkās ("crieurs », de kūknnā «crier» en panjabi) en raison des cris qu'ils poussent lors de certaines pratiques extatiques. L'origine de la secte remonte à la prédication de Balak Singh (c. 1785-1862), de la caste commerçante des Aroṛās dans la région d'Attock, au nord-ouest du Panjab indivis. Le Gurū le plus influent de la secte fut toutefois son successeur, Rām Singh (1816-1885), de la caste d'artisans des Rāmgarhīās, qui en transféra le centre à Bhaini Sahib, dans le district de Ludhiana, au cœur de ce qui est aujourd'hui le Panjab indien. La secte, essentiellement rurale, se forma véritablement autour de lui, rassemblant surtout des Rāmgarhīā et des Jāts d'humble condition. En 1871-1872, les Nāmdhārīs, végétariens, se lancèrent dans une violente campagne contre l'abattage des bovins et s'en prirent aux bouchers musulmans. La répression exercée par les Britanniques se solda par des dizaines d'exécutions au canon, et Rām Singh fut déporté sans jugement à Rangoon en Birmanie, où il finit ses jours. Son jeune frère Hari Singh lui succéda, et fut lui-même assigné à résidence à Bhaini Sahib jusqu'à sa mort en 1906. À cette date, et alors que le fils de Hari Singh, Partāp Singh (m. 1961) devenait le Gurū de la secte, les Nāmdhārīs avaient de longue date renoncé au militantisme et s'attachaient à leurs idéaux de piété personnelle et de vie simple et frugale. Sous la direction de leur nouveau Gurū, Jagjït Singh (1921-2013), fils du précédent, ils soutinrent le parti du Congrès lors du mouvement national. Dans l'Inde indépendante, ils prirent une part active au mouvement pacifique qui aboutit au redécoupage du Panjab indien en 1966 sur 
une base officiellement linguistique (mais en réalité destiné à permettre aux sikhs d'être majoritaires dans le nouvel État). Mais ils se montrèrent hostiles à toute action violente, notamment entre 1980 et 1992, lors de la campagne terroriste des fondamentalistes sikhs qui s'en prirent violemment à eux et dont l'activité tourna à une guerre civile larvée faisant quelque vingt-cinq mille morts au Panjab.

Cette histoire, rapportée en grand détail par Joginder Singh dans l'introduction de son livre, appelle ici deux remarques. D'une part, et cela est d'intérêt pour le sociologue et l'historien des religions, on observe que la succession à la tête de la secte devient rapidement une affaire familiale, et bientôt de père en fils (les intérêts en termes de contrôle des ressources, de pouvoir et de prestige sont souvent considérables, localement, régionalement, nationalement, voire mondialement) : tel est régulièrement le cas dans l'univers sectaire hindou, et tel fut aussi le cas pour la lignée des dix Gurūs des sikhs orthodoxes ${ }^{3}$. D'autre part, et dans le même sens, l'histoire ne s'arrête pas où l'auteur la laisse. Alors même qu'il écrivait son livre, le Gurū nāmdhārī Jagjīt Singh décédait, sans fils ; or, les femmes ne peuvent prétendre au statut de Gurū chez les Nāmdhārīs. Son neveu Udai Singh (né en 1958) était alors choisi pour lui succéder par la partie de la famille contrôlant le siège de la secte, dont la veuve du maître décédé - décision aussitôt contestée par une autre branche de la famille, qui souhaitait imposer un autre neveu du défunt, Dalīp Singh (né en 1953). Le conflit continue, régulièrement marqué par des affrontements violents entre les partisans des deux neveux.

Après ce rappel historique, l'auteur expose sa méthodologie. Afin d'étudier - ce sont les quatre chapitres de son livre - l'« ordre social » des Nāmdhārīs, les effets sur eux de la Partition de 1947 en termes de démographie, les phénomènes de migration et de diversification professionnelle qui ont joué dans l'histoire récente ainsi que la foi et les pratiques religieuses de Nāmdhārīs, l'auteur a constitué un corpus de sources. Ce dernier consiste d'une part en documents d'archives et d'autre part en réponses à un questionnaire de 18 questions (donné en annexe) que Joginder Singh a soumis à 1545 familles de Nāmdhārīs vivant dans 150 villages et 48 villes de 18 des 22 districts du Panjab. Les questions vont de l'identité au style de vie et aux activités sociales et politiques, en passant par l'habitat, la dette éventuelle, l'histoire migratoire de la famille, les relations de caste et les pratiques religieuses. Il résulte de son enquête une image particulièrement claire et complète des Nāmdhārīs aujourd'hui et de certains aspects de leur histoire depuis 1947, ainsi que des données sur leurs pratiques religieuses.

Dans le premier chapitre, la sociologie historique de Joginder Singh lui permet une appréciation fine de la composition de la communauté nāmdhārī en termes de castes. À eux seuls, les Jāts et les Rāmgaṛhāās en représentent 88 \%, et si le taux de conversion est en baisse chez les Jāts, il est en hausse chez les Rāmgaṛhīās. D'une manière générale, les Nāmdhāris recrutent presque exclusivement parmi les castes d'agriculteurs (55\%), Jāts principalement, et d'artisans (c. 40 \%, surtout des Rāmgaṛhīās), et, convient-il d'ajouter, parmi leurs segments les plus défavorisés. L'auteur rapporte l'attrait exercé par la version nāmdhārī du sikhisme sur ces groupes à la dimension égalitaire du code nāmdhārī (Nāmdhārī maryādā) élaboré par le Gurū Rām Singh, qui, en outre, encourage les mariages collectifs pour réduire les dépenses, préconise le recours aux arbitrages locaux pour régler les conflits, notamment en matière de dettes, et exige un mode de vie frugal et végétarien, sans tabac ni alcool, ainsi que le port exclusif de vêtements faits de coton filé à la maison. 

les autres sikhs, les Nāmdhārīs furent pris dans la tourmente des migrations, les sikhs et les hindous fuyant la partie du Panjab appelée à devenir pakistanaise, et les musulmans celle qui allait faire partie de l'Union indienne. Concernant les Nāmdhārīs, 80 \% de ceux qui passèrent à l'est étaient originaires de la seule région de Sialkot. À en croire l'auteur, leur Gurū les encouragea à un départ précoce, ce qui épargna bien des vies, et il pourvut de diverses manières à leur établissement dans leur nouveau pays, achetant ici des terres, et persuadant là tel radjah ou le gouvernement de leur en accorder, notamment parmi celles que des musulmans avaient abandonnées. Tant pour les agriculteurs que pour les artisans, la solidarité de caste et de religion joua aussi un grand rôle : les Nāmdhārīs vivant déjà sur place se montrèrent particulièrement solidaires avec des arrivants qui avaient tout perdu. L'auteur remarque en passant que nombre de familles des castes d'artisans s'établirent auprès des familles de castes d'agriculteurs au service desquelles elles travaillaient avant la Partition.

L'histoire des migrations se poursuit au chapitre suivant, qui traite également de la diversification professionnelle. L'auteur commence par noter que nombre de Nāmdhārīs, comme d'autres Indiens, quittèrent la zone frontalière avec le Pakistan où ils avaient initialement trouvé refuge pour des districts plus orientaux du Panjab - cela en raison de l'exposition de cette partie du territoire indien lors des guerres entre les deux grands pays d'Asie du Sud (1947, 1965-66 et 1971). Un autre choc majeur pour les Nāmdhārīs vint du mouvement de fondamentalisme terroriste évoqué plus haut. En effet, les Nāmdhārīs, à la différence des sikhs orthodoxes, estiment que la lignée des Gurūs humains a continué après Gobind, et que ce dernier ne serait pas mort des coups que lui portèrent deux Pathāns en 1708, mais aurait vécu assez longtemps pour faire de Balak Singh son successeur et se réincarner ensuite dans la personne du Gurū Rām Singh. En raison de cet écart, la terreur s'abattit sur les Nāmdhārīs dans les villages : nombre d'entre eux furent molestés, tués ou contraints à se convertir à la version orthodoxe du sikhisme. Pour sauver leur vie, de nombreuses familles nāmdhārīs quittèrent alors les campagnes où elles vivaient pour aller commencer une nouvelle vie dans les grandes villes du Panjab.

Après ces considérations historiques, et avant d'en venir aux changements de profession, l'auteur s'arrête sur la question, dramatique en Inde, notamment dans les campagnes, de la dette, et présente les statistiques suivantes, selon une grille fondée sur la surface des propriétés terriennes analogue à celle des recensements indiens : $8,42 \%$ des paysans « marginaux » $(28 \%$ du total) sont endettés, $10,49 \%$ des « petits » paysans ( $26 \%$ du total), $8,03 \%$ des « moyens » paysans ( $32 \%$ du total) et $3 \%$ des « gros » paysans ( $14 \%$ du total). La moyenne des paysans nāmdhārīs endettés est donc de 30 \% environ, ce qui est deux fois mieux que la moyenne de l'ensemble des paysans du Panjab, dont $60 \%$ sont endettés.

Même si les Nāmdhārīs restent très majoritairement ruraux, nombre d'entre eux ont migré vers les villes, soit pour les raisons de sécurité évoquées plus haut, soit pour y accéder à des emplois plus lucratifs, et le pourcentage de migrants est nettement plus élevé parmi des artisans que parmi des agriculteurs. Le succès rencontré dans leurs nouvelles activités (professions libérales, enseignement, commerce, industrie, affaires, transport) a entraîné des changements de classe sociale pour de nombreuses familles nāmdhārīs, et c'est désormais au sein de ces nouvelles classes que se recrute la plus grande partie du personnel qui dirige et gère le quartier général de la secte à Bhaini Sahib. 

grande importance au végétarisme des Nāmdhārīs et à leur abstinence de l'alcool et du tabac, qui sont, comme on l'a vu, des prescriptions du code établi par leur deuxième Gurū, suivies selon l'auteur par $98 \%$ des fidèles. $80 \%$ d'entre eux s'abstiennent même de consommer de la nourriture préparée par des non-Nāmdhārīs. Selon Joginder Singh, la vie saine qui résulte de ces pratiques ainsi que les injonctions de vie simple et frugale, l'interdiction de la pratique de la dot - qui endette et ruine nombre de familles indiennes - et le relativement faible taux d'endettement des $\mathrm{Na}^{-}{ }^{-} \mathrm{mha}^{-}$r\&inodot; ${ }^{-} \mathrm{s}$ expliqueraient la quasi-absence de suicide parmi eux, notamment parmi les petits paysans (alors que le suicide parmi ceux des autres communautés, notamment en raison de l'endettement, est un fléau au Panjab comme dans d'autres régions de l'Inde).

obligations religieuses des Nāmdhārīs sont simples. Quotidiennement, ils sont seulement censés se lever à l'aube, se laver puis méditer longuement sur le Nom divin. Il est aussi attendu des familles qu'elles organisent de temps à autre des lectures complètes de l'Ādi Granth, qu'elles fréquentent les lieux de rassemblement communautaire appelés dharamsālās et qu'elles aillent régulièrement « rendre hommage » au Gurū à Bhaini Sahib. Les Nāmdhārīs arborent un rosaire de bois, symbole - selon l'auteur - de leur pacifisme et de leur attachement à la non-violence prônée par Gandhi. Ils vénèrent et, pour beaucoup d'entre eux, lisent régulièrement les livres sacrés du sikhisme, Ādi Granth et Dasam Granth (ouvrage composé dans l'entourage du dixième Gurū et compilé dans sa forme actuelle en 1734).

30 Concernant les sacrements, les cérémonies sont censées être très simples : l'attribution de son nom à un enfant s'accompagne d'une simple lecture de passages de l'Ādi Granth, et en théorie, on n'offre pas de cadeaux. Les mariages, comme il a été dit, sont souvent collectifs, et le rituel central consiste pour les époux à tourner quatre fois autour de l'Ādi Granth dans le sens des aiguilles d'une montre tandis que sont lus par un officiant et répétés par l'assistance les vers d'un hymne du quatrième Gurū des sikhs, Rām Dās (1534-1581). Cette forme de mariage fut initialement légalisée par les Britanniques en 1909 après une intense campagne des réformistes de la Singh Sabhā, soucieux d'avoir pour les sikhs un rituel de mariage différent de celui des hindous. Chez les Nāmdhārīs, il n'y a en théorie, nous l'avons vu, pas de dot (en pratique, cette injonction semble respectée par $40 \%$ des familles), et pas non plus d'échanges de cadeaux lors de la noce.

31 Du mariage, l'auteur en vient au statut des femmes. Selon ses statistiques, le meurtre des filles avant ou après la naissance est moins fréquent parmi les Nāmdhārīs que chez les autres Panjabis : on dénombre 906 femmes pour 1000 hommes parmi les Nāmdhārīs, contre une moyenne de 880 pour 1000 dans le Panjab. Dans quatre districts toutefois (Kapurthala, Tarn Taran, Mohali et Chandigarh), il n'y a parmi les Nāmdhārīs, dans la tranche d'âge 0-6 ans, qu'une moyenne de 770 filles pour 1000 garçons. Et d'une manière générale, alors que plus de filles que la moyenne panjabie savent lire et écrire parmi les Nāmdhārīs, seule une proportion d'entre elles nettement inférieure à la moyenne panjabie accède à des emplois qualifiés - phénomène que l'auteur rapporte aux manques de facilités dans les zones rurales où vit la majorité des Nāmdhārīs. L'égalité entre hommes et femmes est toutefois revendiquée par les Nāmdhārīs, et les filles sont, comme les garçons, initiées dans le Khālsā. Les femmes participent même à l'administration de la secte, jusqu'au niveau central, et le Gurū Rām Singh en nomma une pour le représenter dans une des provinces de sa juridiction spirituelle. Joginder Singh conclut toutefois ce chapitre en insistant sur l'inadéquation de plus en plus ressentie par les Nāmdhārīs entre 
leur code et le «style de vie moderne » : seulement $22 \%$ des familles de son échantillon disent respecter ce code scrupuleusement, pour $67 \%$ partiellement et $11 \%$ pas du tout. Dans la conclusion de l'ouvrage, il attribue à ce décalage grandissant la baisse de $30 \%$, depuis le milieu du xx ${ }^{e}$ siècle, du taux de conversion parmi les sikhs à la version nāmdhārī de leur religion, ainsi qu'au rejet par les idéologues nāmdhārī de la culture populaire panjabie en voie de mondialisation (cinéma, danse, chanson).

Cette première étude d'ensemble sur les Nāmdhārīs est enrichie, en chacun de ses chapitres, de tableaux statistiques issus du traitement des réponses au questionnaire de l'auteur. Mais il est malheureusement dépourvu d'illustrations. On peut remarquer aussi qu'affleure souvent, dans le ton et à travers certains éloges des Nāmdhārīs et de leurs Gurūs, de leurs règles et de leurs pratiques, une empathie qui a peut-être prévenu Joginder Singh, détenteur de la chaire Nāmdhārī Gurū Rām Singh de l'Université Guru Nanak Dev (Amritsar), d'étudier de plus près la vie financière de la secte et celle de son siège à Bhaini, la structure de son pouvoir central et les modalités de contrôle de ses lieux de culte et de leurs revenus.

Les trois ouvrages présentés dans cette note forment, pour qui s'intéresse aux sikhs et au sikhisme ou est engagé dans la recherche à leur sujet, un ensemble de tout premier ordre : le manuel de Louis Fenech et Pashaura Singh est désormais un indispensable outil de référence, et les livres d'Opinderjit Kaur Thakar et de Joginder Singh constituent une excellente introduction à l'étude des sectes dans le sikhisme, une vue d'ensemble étant suivie par une étude de cas très fouillée.

\section{NOTES}

1. Ces notes sont parues respectivement dans Denis Matringe, Les sikhs: histoire et tradition des "Lions du Panjab", Paris, Albin Michel, 2008, p. 327-333, et dans Archives de Sciences Sociales des Religions, 118, 2002, p. 111-114 et 164, 2013, p. 95-101.

2. Comme il est de règle dans les études sikhes, j'appelle "orthodoxes » les continuateurs du courant de réforme qui, à la fin du XIX et au début du $\mathrm{Xx}^{\mathrm{e}}$ siècle, parvint à imposer comme dominante sa vision du sikhisme et de l'identité sikhe (sur ce courant appelé « Tat Khālsā », voir plus bas).

3. Pour un cas hindou, voir Catherine Clémentin-Ojha, «Claiming Religious Rights from a Secular Power. Judgment regarding the Rules of Succession to the Position of Shebait in a North Indian Temple ", in Daniela Berti, Gilles Tarabout and Raphaël Voix (dir.), Filing Religion. State, Hinduism, and Courts of Law, New Delhi, Oxford University Press, à paraître en 2015. 


\section{AUTEURS}

DENIS MATRINGE

CNRS (Centre d'Études de l'Inde et de l'Asie du Sud,UMR 8569 EHESS-CNRS), DMatringe@gmail.com 